\title{
Modelo estructural de competencia profesional didáctica para profesores técnicos no pedagogos
}

Structural Model of Didactic Professional Competency for Non-Educator Technician-Teachers

Artículo de investigación | Research Article

Fecha de recepción: 29 de junio de 2018 Fecha de aceptación: 11 de abril de 2019 Fecha de disponibilidad en línea: marzo 2020

doi: 10.11144/Javeriana.m13.mecp

Marcela Rosa Romero-Jeldres marcela.romero@umce.cl Universidad Metropolitana de Ciencias de la Educación, Chile (iD) ORCID: https://orcid.org/0000-0002-9706-9148

TARIK FAOUZI-NADIM tfaouzi@ubiobio.cl Universidad del Bío-Bío, ChILE ORCID: https://orcid.org/0000-0003-3497-050X

Para citar este artículo | To cite this article Romero-Jeldres, M. R. \& Faouzi-Nadim, T. (2020). Modelo estructural de competencia profesional didáctica para profesores técnicos no pedagogos. magis, Revista Internacional de Investigación en Educación, 13, 1-22. doi: 10.11144/ Javeriana.m13.mecp 


\title{
Resumen
}

Este artículo indaga sobre las competencias pedagógicas de los profesores técnicos sin formación pedagógica que apoyan las trayectorias laborales y educativas de los estudiantes que eligen la educación media técnico profesional (EMTP) en Chile. Utilizando un cuestionario sobre las percepciones acerca del dominio de competencias pedagógicas y la técnica de modelos estructurales, se estudió el dominio asociado con las competencias profesionales didácticas en una muestra estratificada de 151 docentes. Los resultados señalan que el modelo es sensible a los contextos con vulnerabilidad social y al actuar competencial de docentes sin formación, por lo que resulta un aporte para determinar perfeccionamientos focalizados.

\section{Palabras clave}

Competencias del docente; educación técnica; formación de docentes; modelo de ecuaciones estructurales; modelo estadístico

\begin{abstract}
This research delves into the pedagogical competencies of technician-teachers with no pedagogical formation who will support the working and educative trajectory of students who decided to study the Technical-Professional Intermediate Education (EMTP) in Chile. By using a perception questionnaire of the pedagogical competency command as well as the structural model technique, the command of the didactic professional competencies is studied in a stratified sample of 151 teachers. The results indicate that model is sensitive to the contexts with social vulnerability and to the competencybased action of teachers with no formation, which becomes a contribution to determine the focused continuing courses.
\end{abstract}

\section{Keywords}

Technical education; teacher qualifications; statistical models; teacher education; structural equation model 


\section{Descripción del artículo | Article description}

En este artículo de investigación científica y tecnológica, derivado del proyecto de investigación Competencias predominantes para la acción profesional de los docentes y directivos que se desempeñan en modalidad técnico profesional. Hacia la construcción de una pedagogía para educación media técnico profesional, los autores indagan sobre las competencias pedagógicas necesarias para la acción profesional que predominan en los profesores técnicos sin formación pedagógica. La investigación fue financiada a través del Proyecto Fondecyt de Iniciación n. ${ }^{\circ} 11140650$, dirigido por la doctora Marcela Romero-Jeldres, de la Facultad de Filosofía y Educación de la Universidad Metropolitana de Ciencias de la Educación, como investigadora responsable.

\section{Introducción}

La entrada en funcionamiento de la Política Nacional Docente (Ministerio de Educación, 2016) en Chile establece un trayecto de desarrollo para todos los docentes que ejercen en centros con financiamiento estatal. Ello implica que en los próximos años el magisterio chileno verá ajustadas sus remuneraciones conforme a los recorridos propuestos por el denominado encasillamiento profesional, situación que se torna compleja en el caso de los docentes de Educación Media Técnico Profesional (EMTP), generalmente profesionales o técnicos de nivel superior, autorizados por la normativa vigente para llevar a cabo la docencia en las asignaturas técnicas de la enseñanza media cuando no haya oferta de profesores del área (Larrañaga, Cabezas \& Dussaillant, 2013).

Chile se enfrenta a la falta de formadores en centros de educación técnico profesional. Al respecto, Kis \& Field (2009) señalan que muchos profesores y formadores de EMTP tienen solamente la misma cualificación disciplinar en la que enseñan y ninguna otra. Esta situación está ratificada por Rojas (2015), quien da cuenta de que el $48 \%$ de los más de 18000 profesores que ejercen en el sistema técnico profesional chileno no han cursado la carrera de Pedagogía; es decir que la falta de entrenamiento pedagógico afecta a cerca de la mitad de los profesores.

Arias, Farías, González-Velosa, Huneeus \& Rucci (2015) afirman que los docentes de EMTP en servicio han tenido pocas posibilidades de evaluar la calidad de sus desempeños, pues, aunque existen avances en la materia, las evaluaciones a las que estos son sometidos utilizan principalmente los resultados de pruebas de conocimientos disciplinares y su portafolio pedagógico, sin que exista información sobre el logro de aprendizajes por 
parte de los estudiantes de EMTP. Lo anterior cuestiona los dispositivos de formación de los docentes de EMTP, especialmente cuando en los procesos de evaluación no se establece un diálogo entre dos lógicas diferentes: la de los campos disciplinares de las diferentes profesiones de las que provienen los docentes técnico profesionales y la del campo de la enseñanza, la cual o no está cubierta o lo está de manera sucinta a través de títulos de posgrados en Pedagogía para docentes de EMTP, puesto que en la década de los años 80 se cerraron las carreras que impartían enseñanza técnica debido a la falta de postulantes.

No obstante, el problema educacional de mayor preocupación para los próximos años en Chile radica en que tres de cada cinco estudiantes de los primeros tres niveles socioeconómicos siguen la modalidad técnico profesional en la enseñanza media y, según detalla Sevilla-Buitrón (2012), el $64,7 \%$ de los estudiantes de EMTP proviene de los dos quintiles más bajos de ingreso económico familiar. Por tanto, los liceos técnicos en Chile no solo tienen el desafío de fortalecer la formación pedagógica de los profesores de especialidad, sino también de educar a los estudiantes con mayor nivel de desventaja socioeconómica.

Las esperanzas de los estudiantes cifradas en una movilidad que les permita romper con el círculo de pobreza del que provienen siguen siendo desalentadoras a la hora de enfrentarse con las competencias que demanda el mercado laboral o con los nuevos cambios curriculares, orientados hacia las trayectorias formativas con las que puedan acceder a la educación superior (Ministerio de Educación, 2016), dado que los conocimientos adquiridos una vez terminado el ciclo de formación secundaria no son requisito para el ingreso en los centros de formación técnica (CFT) o en las universidades, de manera que los estudiantes suman otra desventaja para proseguir estudios superiores, la cual incide negativamente en sus transiciones formativas y en las perspectivas de movilidad social.

No obstante lo anterior, el diseño de los nuevos marcos de cualificaciones para el aseguramiento de la educación terciaria en Chile implica hacer frente a las 46 opciones de especialidades que tiene la EMTP chilena, agrupadas en 14 áreas ocupacionales, si se quiere facilitar la progresión de un tipo de grado a otro, para dar crédito a las experiencias y competencias académicas y laborales previas (Organización para la Cooperación y el Desarrollo Económicos, 2009); y al mismo tiempo obliga a una revisión general del sistema terciario chileno, dado que a la fecha solo las universidades chilenas tienen el derecho de otorgar títulos de licenciatura a las 18 profesiones tradicionales y de controlar el derecho de practicar la profesión (Organización para la Cooperación y el Desarrollo Económicos \& Banco Mundial, 2009). 
En consecuencia, los liceos chilenos de EMTP, en su mayoría, siguen ofreciendo solamente las condiciones mínimas para garantizar la calidad de la formación y la satisfacción de los requerimientos de acceso para su posterior inserción laboral temprana. Por esta razón, se cuestiona la formación y capacitación de los docentes y las maneras en que se implementa el currículum en estos liceos, dado que las demandas de formación en EMTP ya no se limitan a saberes y teorías, por lo que se espera del docente un doble esfuerzo: por una parte, que pueda enfrentarse al fenómeno de la formación de técnicos de nivel medio y, por la otra, que lleve a cabo una labor orientadora y pedagógica que le permita potenciar las trayectorias progresivas de los estudiantes en los diferentes niveles de la educación secundaria, con el fin de garantizar la continuación de estudios superiores y la inserción en el campo laboral.

Este artículo, por tanto, pone el acento en los profesores de EMTP y en su falta de cualificaciones técnicas y pedagógicas para facilitar la conexión curricular entre los liceos de EMTP y las universidades chilenas; además de otras debilidades asociadas con la alfabetización, la aritmética y las prácticas de las especialidades técnicas. De este modo, esta investigación busca aportar al estudio de las actuales competencias pedagógicas de los profesores de EMTP y de las que se requiere fortalecer para enfrentar los escenarios sociales de empleabilidad y de progresión requeridos.

\section{Referentes conceptuales}

Para situar las conceptualizaciones e identificar las dimensiones subyacentes a las competencias pedagógicas necesarias para la acción profesional de los profesores de EMTP, se utilizaron como soportes teóricos los enfoques europeos alemanes, atendiendo al modelo de educación dual de formación alemana, implementado en Chile desde 1991. El corpus teórico de síntesis planteado va dialogando con los cambios curriculares chilenos y sus demandas, de modo que se pueda construir un modelo de competencia profesional didáctica, lo que impulsa a revisar la vocación laboral docente, por tratarse de profesionales técnicos sin formación pedagógica.

\section{Concepto de competencia pedagógica}

Gröner \& Fuchs-Brüninghoff (2004, p. 238) aportan al léxico de la formación profesional una perspectiva general del término competencia, referido como "la capacidad de acción de una persona", y la diferencian del concepto de cualificación, al cual relacionan con la idea de "gestionar situaciones concretas generalmente ocupacionales". A lo largo del tiempo, el concepto de competencia se ha ido complejizando, determinado por 
diversas áreas, tales como competencias mediáticas, sociales, personales, interculturales, ecológicas, entre otras. No obstante, los autores señalan que las áreas de competencia relevantes para las capacitaciones son la competencia profesional, la competencia en la acción, la competencia metodológica, la competencia personal/humana y la competencia social.

Ahora bien, para privilegiar la perspectiva pedagógica del concepto de competencia, se establece una distinción entre la definición que hace Reetz (2006, p. 305) en el Diccionario de formación profesional y empresarial, en el cual esta se define como las "capacidades humanas que fundamentan y permiten conductas acordes a una situación determinada", y la planteada por Maurer \& Gurzeler (2005, p. 148) en su Manual de competencias, en el que se entiende como "la capacidad de dirigir los recursos o el potencial disponible hacia un objetivo específico", y con ello "combinar conjuntamente el potencial de manera creativa y funcional". Es decir que las competencias, para los autores mencionados, consistirían en los recursos de individuos y grupos que pueden integrarse en las prácticas sociales como resultado de una aplicación exitosa sostenida y que al final pueden acumularse culturalmente.

En ese sentido, Maurer \& Gurzeler (2005) plantean que, en el campo de la docencia, las competencias son mucho más que cualificaciones certificadas, pues con ellas se puede no solo hacer frente a situaciones o requerimientos complejos o entregar resultados de manera eficiente, sino que permiten realizar el potencial creativo que surge en contextos complejos, de modo que en cada situación se lleve a cabo una acción concreta y en situaciones desconocidas, en caso de ser necesario, hayan posibilidades de solución creativas.

\section{Concepto de competencias para la acción profesional docente}

Ahora bien, para comprender el término acción profesional docente, se presta especial atención al aporte teórico que plantean diferentes autores. Tietgens (citado en Peters, 2004), en cuanto a profesionalismo en la educación de adultos, define la acción profesional docente como el poder utilizar la capacidad para aplicar adecuadamente conocimientos de base amplia, científicamente profundos y, por lo tanto, altamente abstractos en situaciones concretas; o, viceversa, reconocer en estas situaciones qué componentes del saber pueden ser relevantes, en las que resulta importante descubrir el problema general en cada caso.

Erpenbeck \& Von Rosenstiel (2003), en el Manual de medición de competencia en la práctica ocupacional, pedagógica y psicológica, señalan que las competencias de acción profesional serían el fundamento para que una persona se comporte de forma holística y autoorganizada, de modo que 
pueda seguir instrucciones, planes propios o ajenos de manera individual o en un sistema social, dado que comprende la capacidad de activar deliberadamente, mediante motivaciones, emociones y experiencias, las demás competencias, y de integrarlas en su campo de acción. Estos autores subdividen las competencias de acción profesional en: competencias personales, competencias orientadas a la actividad y la implementación, y competencias profesionales y metódicas. Posteriormente, Erpenbeck \& Von Rosenstiel (2007) incorporan a la clasificación previa las competencias sociales, las cuales subdividen en habilidades de comunicación y cooperación, crítica y gestión de conflictos, habilidades de equipo, capacidad de persuasión y empatía.

Una tercera perspectiva, planteada por Jank \& Meyer (2009), afirma que la competencia para la acción profesional docente puede ser dividida en tres subcompetencias: a) competencias de implementación, que corresponden a la capacidad de construir situaciones de aprendizaje que involucren a los estudiantes en los procesos de aprendizaje; b) competencias educativas, entendidas como parte de las competencias de acción, porque no sería posible enseñar sin educar, y por ende se compondrían de la capacidad de involucrar completamente a la persona en el proceso de aprendizaje, de modo que el estudiante deba comprenderse como un aliado en el proceso educativo, y, por último, c) las competencias de comunicación, necesarias para acordar y diseñar las clases en conjunto con estudiantes, colegas, apoderados y supervisores.

Una cuarta perspectiva, planteada por Hortsch \& Kersten (2010), señala que reconocer las competencias relacionadas con el actuar en ámbitos laborales y las calificaciones para esa actividad profesional da lugar a la determinación de la competencia para la acción profesional de una persona, y aclaran que esta competencia posee tres dimensiones: la primera se refiere a las condiciones o requisitos informativo-funcionales (dominio de saberes y conocimientos) para la acción profesional, denominado componente funcional; la segunda al componente metodológico (o también procedimental), el cual implica las disposiciones personales para el actuar profesional (dominio de estrategias y procedimientos), y, finalmente, la tercera, la dimensión del componente social, determina el dominio de capacidades interpersonales para la acción profesional en concordancia con las relaciones y valores sociales.

Ahora bien, durante la fase de preparación de la acción profesional, Roswitha (2004) plantea que la profesionalidad se relaciona con algunos requisitos que deben tenerse en cuenta durante las fases de la preparación de la acción profesional: a) ser previamente consciente de las propias tareas de esta, b) tener una comprensión clara y específica del propio rol profesional y del rol del estudiante, c) tener conocimientos y capacidades específicas, 
y d) situarse en un ethos de acción específico. Roswitha (2004) también recomienda tener en cuenta la complejidad de las tareas profesionales, es decir que debe ser requisito de acción obligatoria para los profesionales la disponibilidad de conocimientos y capacidades generales y específicas, adquiridas a través de una adecuada formación científica y práctica profesional, lo que se garantiza por medio de la formación profesional continua, además del constante intercambio y supervisión entre colegas.

En síntesis, se puede señalar que existe concordancia entre los autores en incorporar el componente social dentro del marco de las capacidades interpersonales, planteado por Hortsch \& Kersten (2010), con las relaciones y valores sociales propuestos por Erpenbeck \& Von Rosenstiel (2007), para actuar comunicativa y cooperativamente con autonomía, es decir, para acoplarse y enfrentarse creativamente con otros, para comportarse de un modo orientado a las relaciones y los grupos, y para desarrollar nuevos planes, tareas y objetivos. En segundo lugar, se hace necesario atender dos consideraciones: la primera, lo que se entiende por pedagogos profesionales, definidos por Arnold (1994) como apoyos para el aprendizaje, es el caso de quienes entienden que su oficio debe ejercerse orientado a los objetivos, de forma metódica, y como tales son personas con y de quienes se aprende algo: ellos saben o pueden hacer algo que otros no pueden y están capacitados para conformar una comunidad de aprendizaje, dos aspectos que, en conjunto, conforman la base de la acción pedagógica; la segunda es el planteamiento de Tietgens (citado por Peters, 2004), quien señala que la competencia profesional, la competencia social y la autocompetencia (entendida también como competencia reflexiva) resultan fundamentales para la profesionalización de los profesores; estos tres tipos de competencias estarían integrados en el concepto de competencia profesional didáctica (CPD).

\section{Competencia profesional didáctica}

Jank \& Meyer (2009) parten de la base de que un profesor debe ser capaz de realizar su trabajo diario autónomamente, creativamente, correcto técnicamente, sobre la base de fundamentos científicos y fundamentado en una ética profesional. Para esto se necesita una competencia didáctica, la cual se desarrolla gradualmente por medio del estudio teórico y del trabajo práctico en campos educativos (o relacionados). A este proceso gradual, los autores también lo denominan socialización ocupacional o profesional (Berufssozialisation bezeichnet) señalando las siguientes distinciones teóricas para configurar este actuar: a) la competencia reflexiva sería la capacidad de vincular teoría y conocimiento práctico, y de generar distancia reflexiva de la propia acción. En el caso de la enseñanza, se condensará en representaciones de esta. b) Las competencias de acción profesional hacen 
referencia a la capacidad de orientar el proceso de aprendizaje-enseñanza hacia ciertos objetivos, considerando las especificaciones curriculares e institucionales, en las cuales formarían parte de estas competencias el dominio de un amplio repertorio de métodos (metodológico) y la disponibilidad y la capacidad de realizar las tareas pedagógicas, entre otras.

Ahora bien, para los autores no sería posible actuar didácticamente sin reflexionar didácticamente, y por tanto señalan que el concepto de CPD es integral (en él se fusionan las tres dimensiones de la praxis: pensar-sentiractuar), es transmitido biográficamente (es un flujo directo de la estructura de personalidad del profesor y es directamente determinado por la trayectoria profesional previa) y puede ser diferenciado y mejorado a través de la ejercitación; sin embargo, también puede ser debilitado a través de los cambios en las condiciones laborales, problemas de salud, aplicación de rutinas nefastas, entre otros.

\section{Modelos de formación para situar la competencia profesional didáctica en EMTP}

En primer lugar, es preciso señalar que los modelos de formación para la docencia y la enseñanza tienen en su base distintas representaciones sobre qué hace a un buen docente y de qué capacidades debe disponer para encontrarle sentido al aprendizaje e impartir una formación de calidad (Hallet, 2006). Por tanto, la búsqueda teórica que presentan se realiza con miras al fortalecimiento de la educación técnica chilena, de modo que se halle un modelo que permita el fortalecimiento profesional de los profesionales técnicos no pedagogos.

La primera selección corresponde al Modelo Didáctico de Práctica-Reflexión, planteado por Wallace (1991, p. 14). Este señala que "cada docente debe recurrir al conocimiento empírico, adquirido de sus experiencias, analizadas constantemente sobre el contexto del conocimiento teórico y comprobado en la propia práctica". El autor manifiesta que la buena docencia siempre va acompañada del aprendizaje constante y que toda enseñanza y práctica docente necesita desarrollo continuo, en el cual una cierta capacidad innovadora resulta ser parte fundamental de las competencias docentes.

Jank \& Meyer (2009), desde el enfoque teórico estructuralista y los enfoques teóricos de desarrollo y teórico-biográficos, explican el concepto de acción profesional de modo tal que la profesionalidad docente se conformaría básicamente de la capacidad para comprender y manejar las contradictorias estructuras básicas del trabajo docente, sin agotarse en esa tarea. Es decir: a) no se debe dirigir nunca una situación de enseñanza-aprendizaje y sus objetivos con base en una situación que no es previsible; b) se deben comprender y observar las contradictorias exigencias; c) es necesario 
soportarlas, a partir de las resultantes y crecientes paradojas, y d) deben trabajarse sus consecuencias junto con los estudiantes, estableciendo una distancia reflexiva con su propio actuar pedagógico.

Por ende, desde una perspectiva teórico-estructuralista y desde los enfoques teóricos de desarrollo y teórico-biográficos, la formación docente y las prácticas profesionales deben estructurarse de modo que los profesores puedan establecer para sí mismos tareas de desarrollo que puedan seguir con perseverancia y con apoyo. Para eso deben definir objetivos determinados, aclarar las condiciones generales y organizar la ayuda necesaria para trabajar las áreas conflictivas en las que se mueve la construcción de competencias profesionales reflexivas y de acción, entendiendo siempre que es importante abordar los retos, pero que no existe una secuencia lógica predeterminada a seguir.

En este modelo, la formación docente y las prácticas profesionales deben ser estructuradas de modo que los futuros profesores puedan establecerse a sí mismos tareas de desarrollo que puedan seguir con perseverancia y con apoyo de sus propios profesores. Para esto deben definirse objetivos determinados, aclarar las condiciones generales y organizar la ayuda necesaria en las siguientes cuatro áreas conflictivas para la construcción de competencias profesionales reflexivas y de acción: a) la formulación de un modelo personal; b) la definición y diferenciación de dos o tres tareas de desarrollo y el trabajo en ellas con perseverancia; c) el análisis consciente de las propias fortalezas y debilidades, de la situación de la clase (grupo, curso) y de las condiciones del trabajo escolar, y d) la aclaración de qué es posible enfrentar individualmente y en qué casos se requerirá de ayuda/apoyo externo.

Un tercer modelo estudiado es el Modelo de Competencias Pedagógicas, planteado por Nieke (2002), basado en cuatro componentes, que no pueden separarse, los cuales demandan una base de ciencias de la educación y ética, y contienen aspectos cognitivos, emocionales y conativos (reguladores de la acción) de vital importancia para la configuración y realización de las competencias profesionales didácticas bajo condiciones laborales. El primer componente, análisis de la sociedad, se sitúa en las tareas educativas que surgen en un contexto social. Estas son definidas por las instituciones sociales o generadas por la evolución o el desarrollo social. Las tareas pedagógicas no se deben resolver o enfrentar de manera aislada en las instituciones educativas, sino siempre insertas en un contexto social y político. El segundo componente, diagnóstico de la situación, se refiere a las acciones específicas en el espacio social, visibilizando de esta manera las condiciones, tendencias y necesidades de los estudiantes. Este componente cuenta con cuatro dimensiones: a) la corporalidad involucrada en la acción educativa (uso de los gestos, contacto visual, apropiación espacial, entre 
otros utilizados durante el proceso de aprendizaje-enseñanza y de acuerdo con el clima de aula), b) el aparato mental (identidad y autoconcepto de los estudiantes), c) el espacio (influencia del medio ambiente y el espacio en la acción educativa) y d) la interacción (secuencia constante de acciones y contraacciones con significado simbólico entre profesores y estudiantes). El tercer componente, la autorreflexión, tiene dos dimensiones: a) el autoconcepto profesional, que implica la búsqueda de los principios rectores (¿quién soy yo como maestro(a)?, ¿qué quiero lograr en mi carrera profesional?, ¿qué máximas orientan mi actuar profesional?, ¿cómo puedo medir el efecto de mis acciones?, etc.) y b) la preocupación/afectación por/de sí mismo, porque el profesor(a) está inevitable y directamente involucrado en la acción profesional. Finalmente, el cuarto componente, la acción profesional, difiere de las acciones cotidianas por su componente científico, es decir que sus fundamentos no son intersubjetivos y que son metodológicamente comprobables.

A la luz de los modelos revisados, se optó por las aportaciones del Modelo de Competencias Pedagógicas, de Nieke (2002), la perspectiva de Erpenbeck \& Von Rosenstiel (2007) con respecto a las competencias de comunicación planteadas y por la acción profesional propuesta por Hortsch \& Kersten (2010), en tanto concuerdan con las relaciones y valores sociales planteados por Erpenbeck \& Von Rosenstiel (2007). La figura 1 da cuenta de los constructos teóricos seleccionados para la construcción de un modelo teórico que permita el diálogo con los parámetros de ejercicio para la profesión docente en Chile establecidos por el Ministerio de Educación (2008) a través del Marco para la Buena Enseñanza (MBE).

\section{Vocación laboral docente}

Ahora bien, dado que esta investigación tiene como sujetos de estudio a los y las docentes que se desempeñan como profesores en liceos de educación media técnica en Chile y que, como se ha dicho, el $48 \%$ de los más de 18000 profesores que ejercen en el sistema técnico profesional chileno no han cursado la carrera de Pedagogía, se consideró relevante construir una dimensión cualitativa denominada vocación laboral docente, de modo que fuera un atributo a evaluar, ya que tener o no tener vocación es muy importante en la discursividad de los docentes. En consecuencia, para su construcción, se tuvieron en cuenta las narrativas vocacionales planteadas por Mórtola (2011), las cuales expresan cómo viven su trabajo los docentes a nivel latinoamericano. Ello permitió distinguir tres núcleos vocacionales: el primero, señalado por Alliaud (1993), desde la cultura pedagógica normalista del maestro o maestra, sitúa hegemónicamente los requisitos del ejercicio de la docencia y sus valores, y los dos siguientes, señalados por Dubet (2010), primero, desde la narrativa profesional, como un modo de acceder 
a la plenitud personal y a la realización en la profesión (pasión por enseñar), y luego busca recuperar el conocimiento del estudiante, desde una escuela masificada, que pide rendición de cuentas (transformación social).

De este modo, la figura 1 da cuenta de las dimensiones establecidas en la categoría de vocación laboral docente, sumada a la propuesta teórica de competencias profesionales didácticas para técnicos no pedagogos, de manera que se construye un modelo teórico ecléctico del constructo de CPD para profesores técnicos no pedagogos.

Figura. 1

Propuesta teórica ecléctica de CPD para profesores técnicos no pedagogos

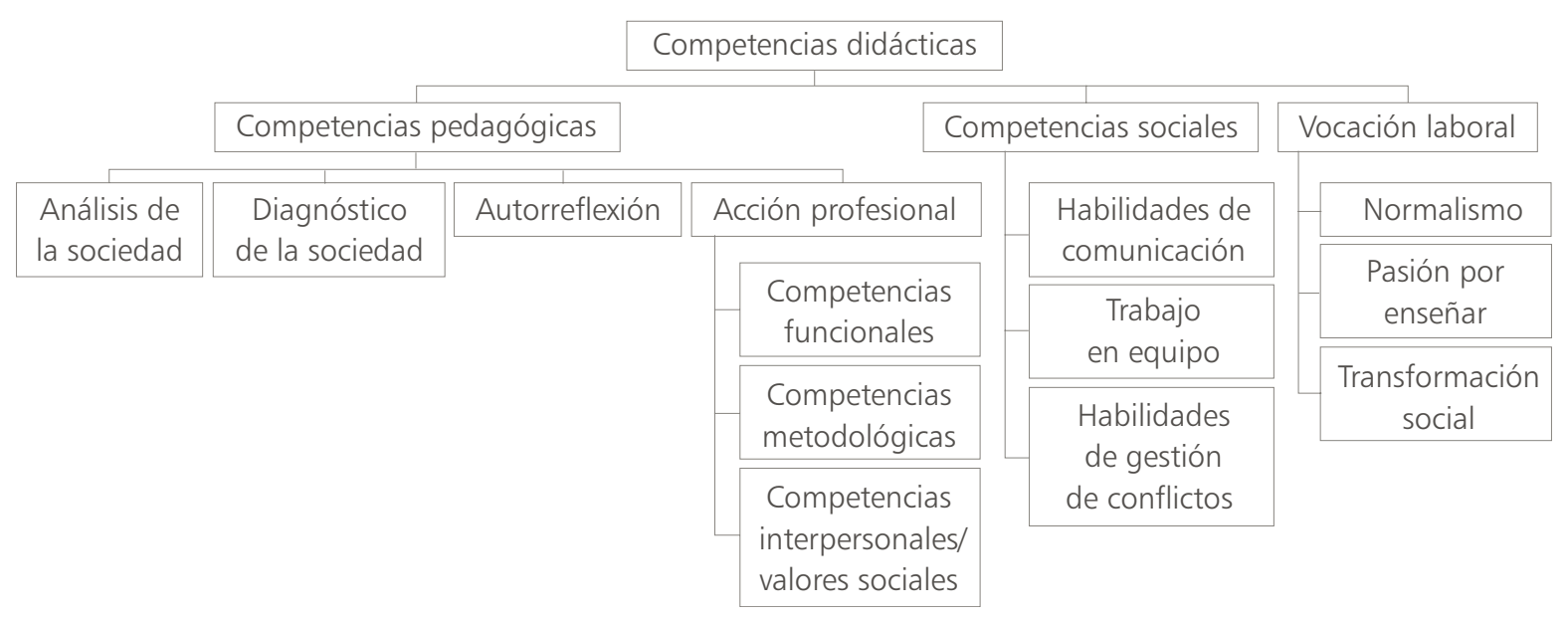

Fuente: elaboración propia

\section{Método}

La investigación se emprendió desde un diseño de investigación mixto concurrente cuantitativo y cualitativo (Hernández, Fernández \& Baptista, 2010), con el fin de indagar en las competencias para la acción profesional que predominan en los docentes y directivos de los liceos de EMTP pertenecientes a la zona sur de Santiago. En este artículo se desarrolla la fase cuantitativa, implementada desde un diseño cuantitativo no experimental multivariante, dada la necesidad de contrastar un modelo teórico que supone relaciones causales entre las variables.

\section{Muestra}

Según los datos filtrados por el Ministerio de Educación (2014), el universo de profesores de EMTP aglutinaba a 234168 profesionales. Por lo tanto, se limitó la población a la organización comunal de la Dirección Provincial Santiago Sur. Ello permitió cubrir 54 liceos de EMTP, con una 
población de 1042 profesores (entre directores y docentes), repartidos en 101 directivos, 173 docentes de especialidad y 768 docentes de formación general. El tamaño de la muestra fue calculado según Hulland, Chow \& Lam (1996), quienes recomiendan una muestra mínima de 100 encuestados. Posteriormente, se estratificó la muestra, por medio de un muestreo aleatorio simple, de modo que quedaron 151 docentes de especialidad y directores, pertenecientes a los 54 liceos existentes en las 9 comunas que atiende la Dirección Provincial Santiago Sur. De estos, el 50,7\% eran hombres y el 49,3\% mujeres; el 57,6\% había asistido a cursos de educación, mientras que el 42,4\% no tenía estudios de este tipo; además, el 71,5\% desconocía el sector económico en el que se clasifica su especialidad en los marcos ministeriales, mientras que el 0,7\% se ubicó en el área agropecuaria y de confección, y el $90 \%$ ejercía la docencia en los programas establecidos en los liceos, mientras que solo un $10 \%$ lo hacía en actividades complementarias o de libre disposición.

\section{Instrumentos}

La información se recogió por medio de un cuestionario estructurado, que utilizó una escala tipo Likert, dirigido a los docentes y directivos docentes de EMTP, construido a partir de las aportaciones teóricas que dieron origen al modelo teórico planteado anteriormente y las variables contextuales, tales como: la comuna donde se lleva a cabo la docencia, el género, la edad, el cargo desempeñado, el tipo de establecimiento, el título profesional, la especialidad en educación, el sector de especialidad en el que realiza la docencia, los cursos que imparten, las horas de contratación, los años de experiencia en educación, los años de experiencia en empresas y el dominio de competencias pedagógicas (Romero-Jeldres \& Faouzi, 2018).

Para la codificación, se utilizó la escala conceptual planteada por Carrera, Vaquero \& Balsells (2011), con la idea de que, a partir de las respuestas de los docentes y los directivos de EMTP, se pudiera reconocer su autopercepción competencial. Se estableció un número determinado de sentencias para cada dimensión teórica, las cuales alcanzaron un total de 126; estas, previa validación de concordancia de cuatro jueces expertos nacionales y cuatro jueces expertos internacionales, se redujeron a 94 sentencias. Para confirmar la validez de contenido, estadísticamente se usó el índice de validez de contenido propuesto por Lawshe (1975), cuyo valor fue de 0,75 . El instrumento se aplicó individualmente en los centros educativos en el período de finalización del año escolar 2016 y a inicios del período escolar 2017, con el fin de evitar que los encuestados tuvieran un exceso de carga laboral. El instrumento se aplicó con un previo consentimiento informado, ajustado a los requerimientos de los comités de ética científica en Chile. 


\section{Procedimiento}

Con los datos recopilados, se realizaron dos fases, de las cuales en este artículo se presenta la segunda.

\section{Análisis de datos}

Los análisis de los datos recabados en la segunda fase establecen una relación causal entre la variable latente competencia didáctica y las variables latentes competencia social, pedagógica y vocacional laboral. Al mismo tiempo, y con el objetivo de calcular el nivel de competencia didáctica (NCD) que poseen los docentes de EMTP, se buscó determinar un índice. Para ello, se comprobó la bondad del instrumento a través de un modelo estructural generado mediante el análisis de ecuaciones estructurales, y se usó el estimador Weighted Least Squares Means and Variance (WLSMV), considerando como variables latentes los factores que componen el instrumento de medida empleado. En este análisis se utilizó el software libre del programa R-Project, versión 3.0.2, y el paquete Latent Variable Analysis (lavaan) (Rosseel, 2012) para hallar el modelo estructural (tabla 1). Para la figura 2, se usó el paquete semPlot (Epskamp, 2015).

\section{Resultados}

\section{Nivel de competencia didáctica (NCD) que poseen los docentes de EMTP: modelo estructural}

Dado que la variable latente competencia didáctica quedó compuesta en el modelo teórico por tres variables exógenas: competencia pedagógica, competencia social y vocación laboral (figura 1), se comprobó la bondad del modelo a través del estimador WLSMV, considerando los ítems como variables categóricas o, cuando la distribución de los datos no fuera normal, utilizando diferentes índices de comprobación de ajuste del modelo (Bentler, 1990; Hu \& Bentler, 1998; McDonald \& Marsh, 1990), los cuales se indican en el párrafo siguiente. Luego de sucesivos ajustes (eliminación de los ítems cuyas contribuciones al modelo resultaran mediocres), el modelo causal surgido de la revisión teórica quedó establecido por dos variables exógenas (competencias sociales y competencias pedagógicas), dejando por fuera la variable vocación laboral docente (figura 2).

Ahora bien, para evaluar la bondad de ajuste, empezando por el modelo teórico hasta llegar a establecer un nuevo modelo (modelo propuesto), se utilizaron diferentes índices: el estadístico chi-cuadrado $\left(x^{2}\right)$, cuyos valores no significativos indican un buen ajuste entre el modelo y los datos empíricos; el comparative fit index (CFI), que toma valores ente 0 y 1 , cuyos valores por encima de 0,90 se consideran como indicadores de un buen 
ajuste entre los datos y el modelo teórico; el root mean standar error of aproximation (RMSEA), que es una medida del grado de error del modelo, cuyos valores por debajo de 0,08 indican un error aceptable, y alrededor de 0,05 indican un muy buen ajuste entre los datos, y, por último, el modelo de Hu \& Bentler (1998), que cumple funciones similares.

Tabla 1

Bondad de ajuste del modelo propuesto

\begin{tabular}{|l|c|c|c|c|c|c|c}
\hline Modelo & $\mathbf{X}^{\mathbf{2}} \mathbf{g} \mathbf{g}$ & $\boldsymbol{p}$-valor & RMSEA & CFI & TLI & $\boldsymbol{\rho}_{\mathbf{v c}}$ & $\boldsymbol{\rho}_{\text {Jor }}$ \\
\hline Modelo teórico & 162,2 & 0,000 & 1,037 & 0,000 & 0,000 & 0,77 & 0,99 \\
\hline Modelo propuesto & 1,07 & 0,163 & 0,022 & 0,999 & 0,999 & 0,61 & 0,97 \\
\hline
\end{tabular}

Figura 2

Nuevo modelo CPD propuesto para EMTP

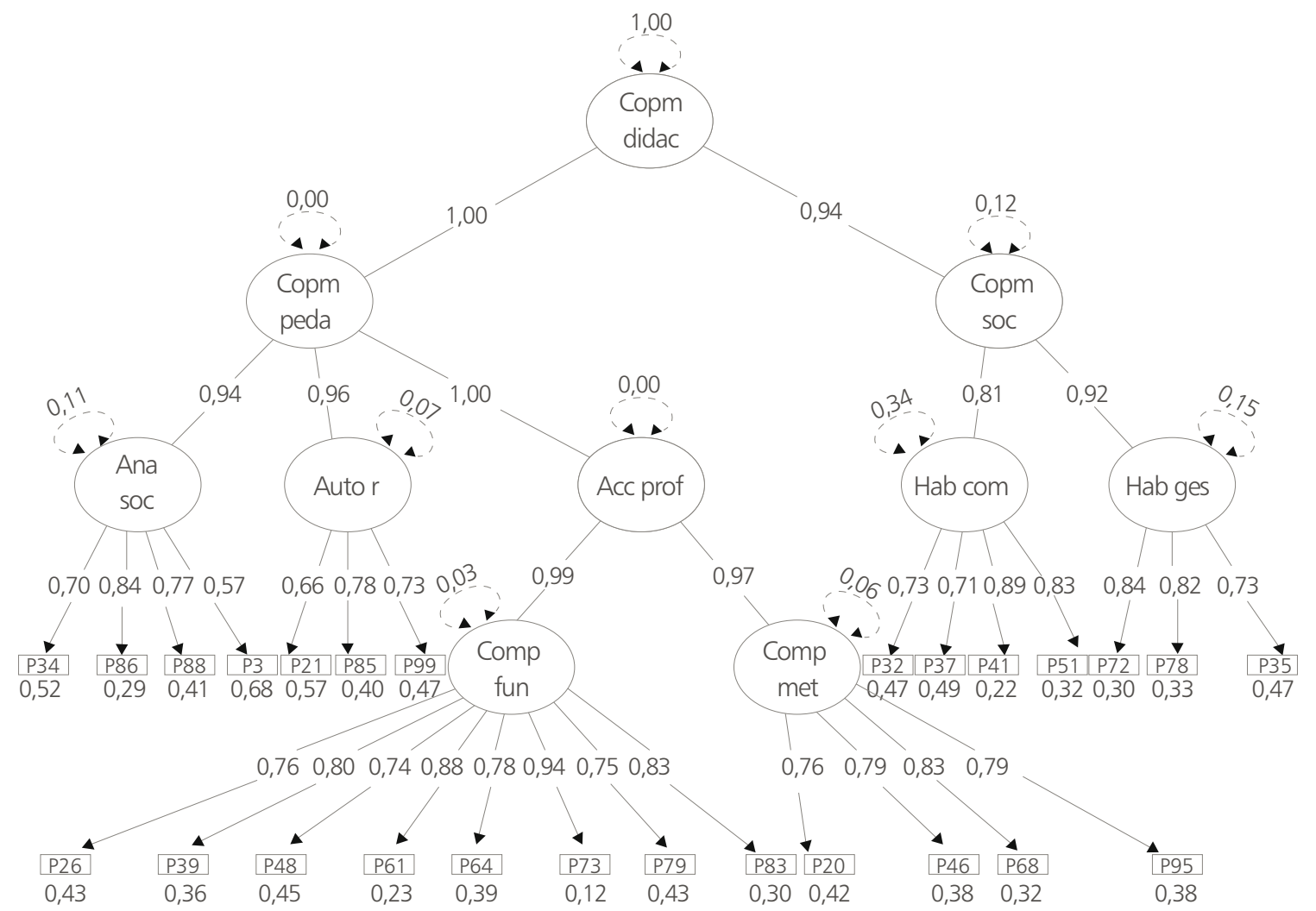


En efecto, la razón entre el estadístico chi-cuadrado y su grado de libertad es inferior a 2, con un p-valor superior al 0,05. El grado de error del modelo tiene un valor de 0,022 , lo cual indica un muy buen ajuste entre los datos y el modelo. Todos los parámetros de los diferentes factores fueron significativos ( $t>1,96)$, por lo que se aceptó el modelo estructural propuesto. Junto a lo anterior, se comprobó la fiabilidad del modelo con el rho ( $\rho)$ de Jöreskog, debido a que este índice es más preciso que el alpha (a) de Cronbach. Según Roussel, Durrieu, Campoy \& El Akremi (2002), el alpha de Cronbach es menos preciso porque es un índice en el que no se consideran los errores en su cálculo.

A través de la fórmula 1, se calculó el pho de Jöreskog, definido por:

$$
\rho_{\text {Jor }}=\frac{\left(\sum_{i=1}^{n} \gamma_{i}\right)^{2}}{\left(\sum_{i=1}^{n} \gamma_{i}\right)^{2}+\sum_{i=1}^{n} \operatorname{Var}\left(\varepsilon_{i}\right)^{2}}
$$

Donde $\gamma_{i}$ es la contribución de la variable latente a la variable observada, $n$ es el número de variables observadas y $\varepsilon_{i}$ es el error de medida. El valor hallado por la ecuación 1 es de 0,97. Para la validez interna del constructo, se usó el índice de validez convergente y se comprobó que los constructos que se esperaba que estuvieran relacionados de hecho lo están. Calculando este índice, definido por la ecuación 2, se obtuvo un valor de 0,61 , considerado satisfactorio.

$$
\rho_{v c}=\frac{\sum_{i=1}^{n} \gamma_{i}^{2}}{\sum_{i=1}^{n} \gamma_{i}^{2}+\sum_{i=1}^{n} \operatorname{Var}\left(\varepsilon_{i}\right)^{2}}
$$

A través de la fórmula 3, usando el índice de Fornell, Johnson, Anderson, Cha \& Bryant (1996), se calculó el nivel de competencia didáctica que poseen los docentes de EMTP (IIISD), para el que se obtuvo un valor de 0,79\%. Este valor indica que los profesores técnicos no pedagogos son siempre capaces de realizar la cuestión propuesta en materia de competencias didácticas, pero que presentan dificultades para explicar o enseñar a otra persona aquello que dicen saber hacer.

$$
\text { IIISD }=\frac{E[\phi]-\operatorname{Min}[\phi]}{\operatorname{Max}[\phi]-\operatorname{Min}[\phi]} \times 100
$$

Donde $\operatorname{Min}[\phi]=\sum_{i=1}^{n} \mathrm{~W}_{\mathrm{i}} \operatorname{Min}\left[\mathrm{p}_{\mathrm{k}(\mathrm{i})}\right]$ y $\operatorname{Max}[\phi]=\sum_{i=1}^{n} \mathrm{~W}_{\mathrm{i}} \operatorname{Max}\left[\mathrm{p}_{\mathrm{k}(\mathrm{i})}\right] ; \phi$ es una variable latente y $E[\phi]$ es la esperanza de la variable $\phi$ expresada como $\mathrm{E}[\phi]=\sum_{i=1}^{n} \mathrm{w}_{\mathrm{i}} \mathrm{p}_{\mathrm{k}(\mathrm{i})} ;$ el $\operatorname{Min}\left[p_{k(i)}\right]$ y el $\operatorname{Max}\left[p_{k(i)}\right]$ son los valores mínimo y máximo, respectivamente, del rango de los ítems. En este caso, $\operatorname{Min}\left[p_{k(i)}\right]=1$ $\mathrm{y} \operatorname{Max}\left[p_{k(i)}\right]=5,0$. 


\section{Discusión de resultados}

En este estudio se han podido comprobar las bondades de los modelos de ecuaciones estructurales como métodos de análisis multivariables para usar simultáneamente una serie de relaciones de dependencia (MejíaPuente \& Cornejo-Sánchez, 2010), lo que les permite a los investigadores en educación tratar variables que en un proceso pueden ser independientes o dependientes y asumir otra relación en el mismo modelo. Por otra parte, al igual que plantea Corral (2002), también fue posible evitar tener que hacer otras pruebas al instrumento para probar la relación entre las variables latentes, dado que la confiabilidad se pudo estimar por medio de los pesos factoriales de cada variable latente, lo que da mayor fuerza explicativa al modelo obtenido.

Con todo, el desarrollo de la investigación no solo permitió proponer la construcción de una base conceptual organizada en un modelo teórico de competencias profesionales didácticas, sino que también hizo posible explicarlo por medio de los datos arrojados por los docentes participantes que ejercen en EMTP. Así, se redujeron y agruparon las variables latentes del modelo teórico de referencia en otro modelo teórico causal competencial para profesores técnicos no pedagogos que considera el desempeño laboral de formación en contextos de alta desventaja social y económica.

Ahora bien, confirmar este modelo causal en contextos de pobreza permite señalar que el dominio de las competencias didácticas profesionales puede explicarse evaluando el dominio de las competencias pedagógicas y las competencias sociales. En el primer caso, las competencias pedagógicas se explican según el dominio que los docentes poseen en dimensiones asociadas con el análisis de la sociedad, la autorreflexión y la acción profesional. Estos requerimientos ratifican la necesidad de poseer un dominio de base en la formación docente y, salvo el descarte de la variable latente diagnóstico social, los resultados validan el Modelo de Competencias Pedagógicas, propuesto por Nieke (2002).

Con respecto al dominio en las competencias sociales, planteadas por Hortsch \& Kersten (2010) y Erpenbeck \& Von Rosenstiel (2007), en concordancia con las disposiciones para actuar comunicativa y cooperativamente de forma autoorganizada, los resultados se explican en relación con las habilidades de comunicación y las habilidades de gestión de conflictos, documentadas en la revisión teórica. De este modo, los resultados planteados en esta investigación resultan muy relevantes en los requerimientos del modelo causal, ya que ello no es explícito en el MBE (Ministerio de Educación, 2008), y por tanto resulta una competencia a desarrollar en la docencia cuando el desempeño laboral docente se lleva a cabo en contextos en los 
que la pobreza extrema, la exclusión y la vulnerabilidad de las familias y estudiantes se integra en los liceos asociados con violencia social y desintegración social. Según Tedesco \& Tenti-Fanfani (2002), ello tendría efectos directos en el trabajo docente, sobre todo cuando se debe cumplir con tareas asistenciales consideradas como socialmente urgentes.

De ese modo, este hallazgo en el modelo causal resulta de la mayor importancia para orientar la formación continua de pedagogos, como en la capacitación de profesores de EMTP que se enfrentan a la formación técnica sin tener formación pedagógica, al tiempo que aporta evidencia empírica que respalda la singularidad competencial requerida por los docentes que se desempeñan en EMTP.

Por otra parte, con base en el índice de Fornell, con los datos suministrados por los docentes de EMTP, se calculó el nivel de competencia didáctica (NCD). El valor satisfactorio obtenido permite explicar el nivel de competencias didácticas desde dos perspectivas diferentes: la primera indica que los docentes de EMTP poseen competencias pedagógicas, pero la segunda muestra que no pueden explicar ni enseñar a otra persona el conocimiento que poseen.

Este índice constituye un aporte para utilizar el instrumento validado en esta investigación con el fin de determinar los requerimientos de capacitación que requieren los docentes que trabajan en la EMTP. Al mismo tiempo, se considera relevante organizar los resultados obtenidos desde las dos perspectivas mencionadas; es decir, por una parte, para identificar el nivel de competencias pedagógicas que poseen los docentes de acuerdo con el modelo causal propuesto y, por otra parte, para tomar conciencia de que las capacitaciones que brindan los organismos técnicos y las universidades deben asegurar no solo el dominio teórico de las competencias, sino también el dominio práctico, puesto que el mayor logro competencial del modelo se juega en la medida en que un docente es capaz de explicar o enseñar lo aprendido.

\section{Conclusiones}

Se puede afirmar que los resultados aportaron: a) la validación de un modelo teórico de competencias profesionales didácticas para profesores técnicos sin formación profesional pedagógica que identifica dominios en competencias pedagógicas adquiridas experiencialmente; no obstante, la falta de formación en pedagogía no les permite ser evaluados con los mismos parámetros establecidos por el Sistema de Desarrollo Profesional Docente chileno; b) el dominio de competencias pedagógicas que los docentes declaran no alcanza el nivel de autonomía necesario para hacer enseñable 
un saber con base en la pedagogía y, por tanto, el carácter del perfeccionamiento docente debe situarse desde el hacer y el actuar profesional, si se quieren fortalecer las competencias profesionales didácticas (esto debe también ser una condición en todos los perfeccionamientos docentes), y c) se considera necesario que la investigación en educación integre en los análisis los modelos de ecuaciones estructurales, con el fin de evidenciar otros fenómenos educacionales cuando se hacen converger múltiples variables; esto favorece la comprensión de los contextos educacionales en su total complejidad, y poco a poco va sepultando la clausura que el ceteris paribus impone a las ciencias sociales (al tener que mantener constantes otras variables, mientras se revisa el efecto de una variable sobre otra) y permite también admitir la no igualdad de los fenómenos en las investigaciones.

\section{Aclaraciones y agradecimientos}

Los resultados de esta publicación han sido posibles gracias al financiamiento otorgado por Conicyt, a través del Proyecto Fondecyt de iniciación n. ${ }^{0} 11140650$.

\section{Sobre los autores}

Marcela Rosa Romero-Jeldres es doctora en Ciencias de la Educación y magíster en Comunicación Social de la Pontificia Universidad Católica de Chile, Chile. Es licenciada en Educación y profesora de Educación General Básica. Además, es profesora titular del Departamento de Formación Pedagógica de la Facultad de Filosofía y Educación de la Universidad Metropolitana de Ciencias de la Educación, Chile.

Tarik Faouzi-Nadim es doctor en Estadística de la Universidad de Valparaíso, Chile, y magíster en Ciencias Tecnológicas y Aplicaciones Matemáticas de la Universidad de Pierre y Marie Curie, Francia. Es licenciado en Matemáticas de la Universidad de Ibn Zohr, Marruecos. Es académico del Departamento de Estadísticas de la Facultad de Ciencias, de la Universidad del Bío-Bío, Chile.

\section{Referencias}

Alliaud, A. (1993). El maestro como categoría social: génesis y desarrollo en Argentina (1880-1915). Buenos Aires: CEAL.

Arias, E., Farías, M., González-Velosa, C., Huneeus, C. \& Rucci, G. (2015). Educación técnico profesional de Chile. Santiago: Banco Interamericano de Desarrollo.

Arnold, R. (1994). Berufsbildung. Annäherungen an eine evolutionäre Berufspädagogik. Berlín: Schneider Verlag Hohengehren. 
Arnold, R. (2006). Selbstorganisation. In F.-J. Kaiser \& G. Pätzold. (Hrsg.). Wörterbuch Berufs - und Wirtschaftspädagogik. 2 überarbeite und erweiterte Auflage (439-440). Bad Heilbrunn.

Bentler, P. M. (1990). Comparative fit indexes in structural models. Psychological Bulletin, 107, 238-246.

Carrera, F., Vaquero, E. \& Balsells, M. (2011). Instrumentos de evaluación de competencias digitales para adolescentes en riesgo social. Edutec, Revista Electrónica de Tecnología Educativa, 35, 1-17.

Corral, V. (2002). Structural equation modeling. En R. Bechtel \& A. Churchman, Handbook of Environmental Psychology (pp. 256-270). Nueva York: John Wiley.

Dubet, F. (2010). Crisis de la transmisión y declive de la institución. Revista Política y Sociedad, 47(2), 15-25.

Epskamp, S. (2015). SemPlot: Unified visualizations of structural equation models. Structural Equation Modeling: A Multidisciplinary Journal, 22(3), 474-483.

Erpenbeck, J. \& Von Rosenstiel, L. (2003). Handbuch Kompetenzmessung. Stuttgart: Schäffer-Poeschel.

Erpenbeck, J. \& Von Rosenstiel, L. (2007). Handbuch Kompetenzmessung. 2. Vorbemerkung zur 2. Auflage. Stuttgart: Schäffer-Poeschel.

Fornell, C., Johnson, M. D., Anderson, E. W., Cha, J. \& Bryant, B. E. (1996). The American customer satisfaction index: Nature, purpose, and findings. Journal of Marketing Research, 60(4), 7-18.

Gröner, H. \& Fuchs-Brüninghoff, E. (2004). Lexikon der Berufsausbildung. Über 1500 Begriffe für Ausbilder, Führungskräfte und Personalentwickler. Múnich: Deutschen Taschenbuch Verlag.

Hallet, W. (2006). Didaktische Kompetenzen. Lehr-und Lernprozesse erfolgreich gestalten. 1. Auflage. Stuttgart: Klett Lernen und Wissen GmbH.

Hernández, R., Fernández, C. \& Baptista, L. (2010). Metodología de la investigación. México: McGraw-Hill Interamericana.

Hortsch, H. \& Kersten, S. (2010). Didaktik der Berufsbildung, Merkblätter. Dresde: SFPS-Wissenschaftlicher Fachverlag.

Hu, L. T. \& Bentler, P. M. (1998). Fit indices in covariance structure modeling: Sensitivity to underparameterized model misspecification. Psychological Methods, 3(4), 424-453.

Hulland, J., Chow, Y. H. \& Lam, S. (1996). Use of causal models in marketing. International Journal of Research in Marketing, 13(2), 181-197.

Jank, W. \& Meyer, H. (2009). Didaktische Modelle. Berlín: Cornelsen Verlag.

Kis, V. \& Field, S. (2009). Learning for Jobs. The OECD Reviews of Vocational Education and Training. Chile: A First Report. París: OECD.

Larrañaga, O., Cabezas, G. \& Dussaillant, F. (2013). Informe completo del Estudio de la Educación Técnico Profesional. Santiago: Programa de las Naciones Unidas para el Desarrollo-Chile, Área de Reducción de la Pobreza y la Desigualdad. Recuperado de http://www.undp.org/content/dam/chile/docs/ pobreza/undp_cl_pobreza_etp_2013.pdf. 
Lawshe, C. H. (1975). A quantitative approach to content validity. Personnel Psychology, 28(4), 563-575.

Maurer, H. \& Gurzeler, B. (2005). Handbuch Kompetenzen. Arbeitsinstrument zur Entwicklung überfachlicher Kompetenzen. Berna: Hep Verlag.

McDonald, R. P. \& Marsh, H. W. (1990). Choosing a multivariate model: Noncentrality and goodness of fit. Psychological Bulletin, 107(2), 247-255.

Mejía-Puente, M. H. \& Cornejo-Sánchez, C. S. (2010). Aplicación del modelo de ecuaciones estructurales a la gestión del conocimiento. Latin American \& Caribbean Journal of Engineering Education, 4(1), 23-30.

Ministerio de Educación. (2008). Marco para la Buena Enseñanza. Recuperado de https://www.cpeip.cl/marco-buena-ensenanza/

Ministerio de Educación. (2014). Base de Datos Oficiales 2014. Santiago: Centro de Estudios. Ministerio de Educación. Recuperado de https://centroestudios. mineduc.cl/wp-content/uploads/sites/100/2017/06/Estad\%C3\%ADsticasde-la-Educación-2014.pdf

Ministerio de Educación. (2016). Política Nacional Docente. Recuperado de https://bibliotecadigital.mineduc.cl/handle/20.500.12365/2162

Mórtola, G. (2011). Breve ensayo sobre la vocación de los maestros y maestras en Argentina. Buenos Aires: Centro Editor de América Latina.

Nieke, W. (2002). Sorgsamer Umgang mit der Natur-Umwelt als Aufgabe der Allgemeinbildung. Rostock: unveröffentl. Ms.

Organización para la Cooperación y el Desarrollo Económicos (OCDE). (2009). Revisiones de la OCDE sobre el mercado laboral y las políticas sociales. Chile. París: OCDE.

Organización para la Cooperación y el Desarrollo Económicos (OCDE) \& Banco Mundial. (2009). Revisiones de políticas nacionales para educación: terciaria Educación en Chile. París: OCDE.

Reetz, L. (2006). Kompetenz. En F. J. Kaiser \& G. Pätzold, Wörterbuch Berufs -und Wirtschaftspädagogik. 2. Auflage. (pp. 305-307). Bad Heilbrunn: Julius Klinkhardt Verlag.

Rojas, R. (2015). Más de 8 mil profesionales no pedagogos podrán seguir haciendo clases en liceos técnicos. Diario Uchile-Radio Universidad de Chile, 16 de mayo. Recuperado de http://radio.uchile.cl/2015/05/16/mas-de-8-milprofesionales-no-pedagogos-podran-seguir-haciendo-clases-en-liceostecnicos/

Romero-Jeldres, M. \& Faouzi, T. (2018). Validación de un modelo de competencias pedagógicas para docentes de Educación Media Técnica. Educación y Educadores, 21(1), 114-132. doi: 10.5294/edu.2018.21.1.6

Rosseel, Y. (2014). The lavaan tutorial. Bélgica: Department of Data Analysis: Ghent University.

Roswitha, P. (2004). Erwachsenenbildungs-Professionalität. Ansprüche und Realitäten. Bielefeld: W. Bertelsmann Verlag.

Roussel, P., Durrieu, F., Campoy, E. \& El Akremi, A. (2002). Méthodes d'équations structurelles: Recherche et Applications en Gestion. París: Economica. 
Sevilla-Buitrón, M. P. (2012). Educación técnica profesional en Chile: antecedentes y claves de diagnóstico. Santiago: Centro de Estudios, División de Planificación y Presupuesto, Ministerio de Educación.

Tedesco, J. C. \& Tenti-Fanfani, E. (2002). Nuevos tiempos, nuevos docentes. Buenos Aires: IIPE-Buenos Aires.

Peters, R. (2004). Erwachsenenbildungs-Professionalität. Bielefeld: W. Bertelsmann Verlag.

Wallace, M. (1991). Training Foreign Language Teachers. A reflective approach. Glasgow: CUP. 